\title{
The unidirectional phylogeny of Homo sapiens anchors the origin of modern humans in Eurasia
}

Úlfur Árnason ${ }^{1,2^{*}}$

\begin{abstract}
Background: The Out of Africa hypothesis, OOAH, was challenged recently in an extended mtDNA analysis, PPA (Progressive Phylogenetic Analysis), that identified the African human populations as paraphyletic, a finding that contradicted the common OOAH understanding that Hss had originated in Africa and invaded Eurasia from there. The results were consistent with the molecular Out of Eurasia hypothesis, $\mathrm{OOEH}$, and Eurasian palaeontology, a subject that has been largely disregarded in the discussion of OOAH.

Results: In the present study the mtDNA tree, a phylogeny based on maternal inheritance, was compared to the nuclear DNA tree of the paternally transmitted Y-chromosome haplotypes, Y-DNAs. The comparison showed full phylogenetic coherence between these two separate sets of data. The results were consistent with potentially four translocations of modern humans from Eurasia into Africa, the earliest taking place $\approx 250,000$ years before present, YBP. The results were in accordance with the postulates behind OOEH at the same time as they lent no support to the OOAH.
\end{abstract}

Conclusions: The conformity between the mtDNA and Y-DNA phylogenies of Hss is consistent with the understanding that Eurasia was the donor and not the receiver in human evolution. The evolutionary problems related to OOAH became similarly exposed by the mtDNA introgression that took place from Hss into Neanderthals $\approx 500,000 \mathrm{YBP}$, a circumstance that demonstrated the early coexistence of the two lineages in Eurasia.

Keywords: Human evolution, Molecular phylogenetics, Out of Eurasia hypothesis, OOEH, Out of Africa hypothesis, OOAH, mtDNA, nuDNA, Y-DNA

\section{Background}

A continuous unidirectional evolution from the root of the tree to the tip of each individual branch is an indisputable requirement for the validity of any phylogenetic tree. In a recent study [1] the direction of evolution in the tree of modern man, Homo sapiens sapiens, Hss, and man's closest extinct relatives was determined in accordance with this condition. The results were inconsistent

\footnotetext{
*Correspondence: ulfur.arnason@gmail.com; ulfur.arnason@med.lu.se ${ }^{1}$ Department of Clinical Sciences Lund, Lund University, Lund, Sweden Full list of author information is available at the end of the article
}

with the common OOAH understanding that Hss had originated in Africa and invaded Eurasia from there.

In their study [1] the authors introduced a new molecular approach, Progressive Phylogenetic Analysis (PPA) that allowed establishment of the direction of evolution in the Hss tree. The results showed that the African Hss populations constituted a paraphyletic assembly, a finding that compromised the foundation of the OOAH. In comparison the established PPA phylogeny was consistent with the OOEH and the Eurasian palaeontology of both $H s s$ and $H s n(H s n n+H s n d)$, a topic that has been largely ignored by the adherents of OOAH.

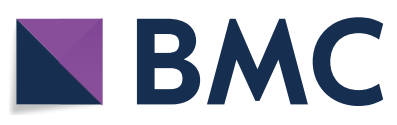

(c) The Author(s) 2021. Open Access This article is licensed under a Creative Commons Attribution 4.0 International License, which permits use, sharing, adaptation, distribution and reproduction in any medium or format, as long as you give appropriate credit to the original author(s) and the source, provide a link to the Creative Commons licence, and indicate if changes were made. The images or other third party material in this article are included in the article's Creative Commons licence, unless indicated otherwise in a credit line to the material. If material is not included in the article's Creative Commons licence and your intended use is not permitted by statutory regulation or exceeds the permitted use, you will need to obtain permission directly from the copyright holder. To view a copy of this licence, visit http://creativecommons.org/licenses/by/4.0/. The Creative Commons Public Domain Dedication waiver (http://creativeco mmons.org/publicdomain/zero/1.0/) applies to the data made available in this article, unless otherwise stated in a credit line to the data. 


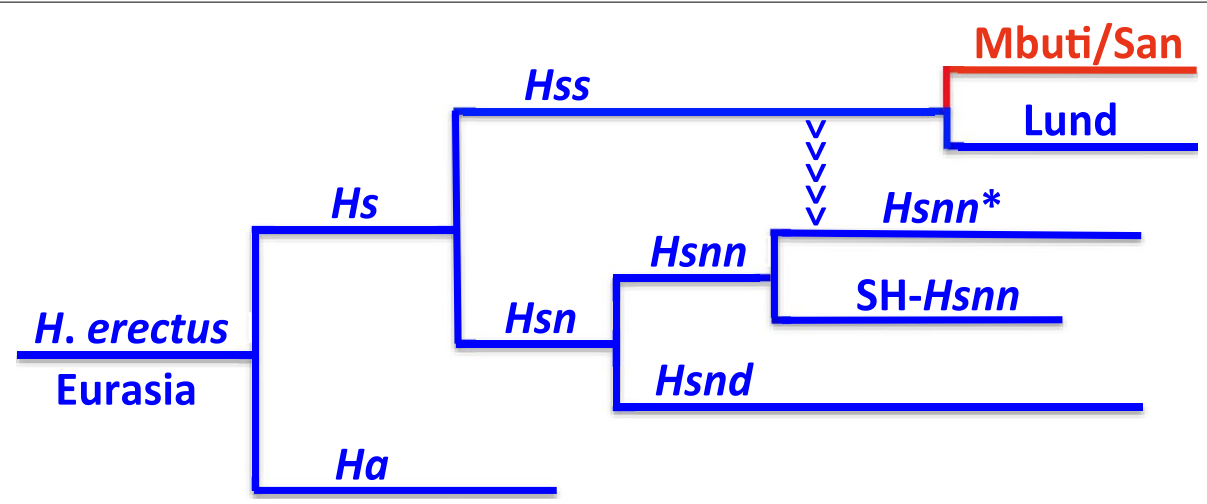

Fig. 1 The nuDNA phylogeny leading to Hss, Homo sapiens sapiens. Blue: Eurasian lineages. Red: African lineages. H. erectus has been placed at the root of the tree in accordance with the artefact sequence related to the Eurasian existence of $\mathrm{He} 2,12 \mathrm{MYBP}$ [15]. The divergence between $\mathrm{Hs}, \mathrm{H}$. sapiens, and $\mathrm{Ha}, \mathrm{H}$. antecessor, has been dated to $\approx 850,000 \mathrm{YBP}[16,17]$, that between $\mathrm{Hss}$ and Hsn, H. s. neanderthalensis, to $\approx 800,000 \mathrm{YBP}$ and that between Lund and Mbuti/San to $\approx 250,000$ YBP. Hsn divides into Hsnn, Neanderthals proper, and Hsnd, Denisova, with Hsnn dividing further into $\mathrm{SH}-\mathrm{Hsnn}$ (Hsnn at Sima de los Huesos) and Hsnn*, a branch arising as the result of the mtDNA introgression that took place from Hss into Hsnn* $\approx$ 500,000 YBP [1]

Here the evolution of Hss was addressed in the light of the phylogenies of two separate sets of data, viz. the nuclear DNA, nuDNA, tree of the paternally transmitted Y-chromosome haplotypes, Y-DNAs [2-4], and the mtDNA tree, a non-nuclear tree with maternal inheritance. The findings supported conclusively the results presented in the initial Y-DNA studies $[3,4]$ and the more recent mtDNA findings that have challenged OOAH $[1$, $5,6]$.

During the latter part of the 1980s, fragmentary sequence data from mtDNA became introduced in molecular phylogenetics with the aim of establishing the relationships within and among different species. In this era the group of Allan C. Wilson presented results that, according to the authors $[7,8]$, provided evidence that $H s s$ had originated in Africa and subsequently migrated from there into Eurasia. The hypothesis soon became the norm in the discussions of Hss evolution, although the molecular basis for the understanding was questionable and the palaeontological support for it was lacking. Furthermore, examination of the same data in other molecular studies [9-11] did not favour the trees upon which the OOAH postulate was proposed.

The extensive studies of human $Y$ chromosome haplotypes referred to above $[3,4]$ have yielded comprehensive phylogenetic results that are highly relevant to the discussion of OOAH. Contrary to the common OOAH understanding these studies identified a basal divergence between the non-African and African Hss populations, a finding that was incompatible with the OOAH position of a late Hss exodus out of Africa.

Here the topologies of the Y-DNA tree $[2,4]$ and the PPA phylogeny of the mtDNA tree of Hss [1] were compared. Thus, the comparison comprised two evolutionarily separate sets of data, viz. a paternal nuclear marker, Y-DNA, and a non-nuclear marker, mtDNA, based on maternal inheritance. The comparison yielded results that were mutually consistent with each other and with the OOEH while they lent no support to the out of Africa hypothesis.

The Y-DNA evolution of Neanderthals and Denisovans was addressed in a recent study [12] that related the Neanderthal mtDNA replacement to an ancient gene flow from an early lineage related to modern humans. The authors [12] referred to two studies [13, 14] in this connection, both acknowledging Hss origin in Africa and OOAH. One of these studies [13] presented a comprehensive OOAH account that rested upon $H s$ origin in Africa followed by an African divergence between Hss and $H s n$ (Neanderthals/Denisovans). This stage was followed by an out of Africa exodus of $H s n$ and a Eurasian divergence of $H s n$ into Neanderthals and Denisovans and a late out of Africa exodus of Hss. These circumstances have limited support in palaeontology and molecular findings related to $H s(H s s+H s n)$ evolution.

\section{Results and discussion}

The out of Eurasia phylogeny

Figure 1 outlines basal $H s$ relationships in accordance with recent advances in Eurasian palaeontology and phylogenetics [1]. The Eurasian-derived parts of the figure are marked blue and the African contribution red. Homo erectus, $\mathrm{He}$, has been placed at the root of the tree consistent with $\mathrm{He}$ entering Eurasia from Africa $>2$ million years ago [15] in agreement with the Eurasian palaeontology of 


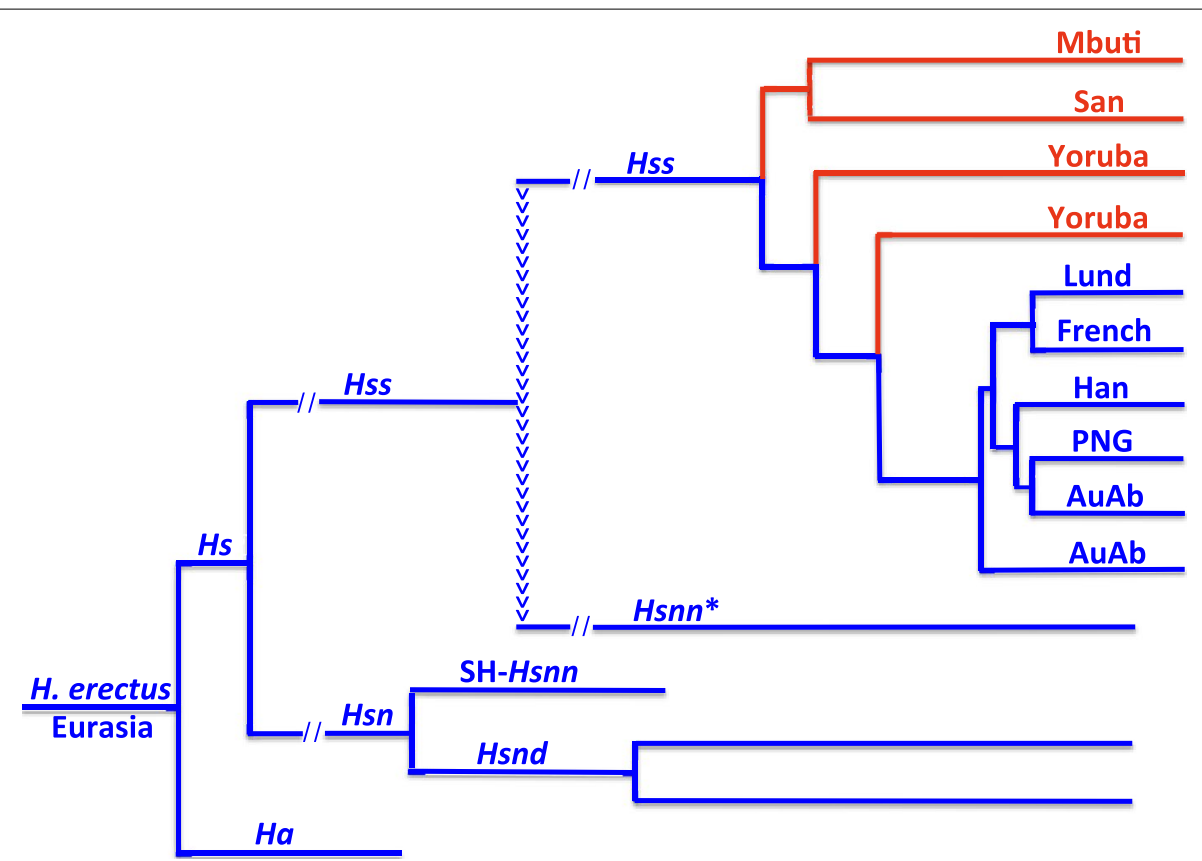

Fig. 2 The mtDNA relationships of Hs demonstrating the paraphyly of the African Hss populations as resolved by PPA. Blue: non-African taxa; red: African taxa. Hsnn*: Hsnn other than SH-Hsnn. The arrowheads signify the mtDNA introgression that gave rise to Hsnn*. The limitation of Hsnn to Eurasia places the mtDNA introgression in this continent, reversing the direction of Hss evolution behind OOAH. The Hss part of the tree underlines the phylogenetic continuity among non-African populations and the paraphyly of the African populations including the two Yoruba [1]. AuAb: Australian aborigines; PNG: Papua New Guinean; Han: Chinese; Lund: The first described non-chimaeric human mtDNA molecule [19]; French: A European, as representing previous genomic findings [20]

the species. As apparent the evolution is continuous and unidirectional through the entire tree from the oldest to the most recent Hss divergence as represented by the African Mbuti/San and the Eurasian Lund.

The earliest divergence in Fig. 1, that between $\mathrm{Ha}$, Homo antecessor, and Hs, Homo sapiens, has been placed palaeontologically [16] and molecularly [17] at $\approx 850,000$ YBP. As Fig. 1 shows, the $H s$ branch splits into a branch leading to extant humans, Hss, and another branch, $H s n, H$. sapiens neanderthalensis, that gave rise to Hsnn, Neanderthals proper, and Hsnd, Denisovans. Hsnn diverged early into two branches, $\mathrm{SH}-H s n n$ and $H s n n^{*}$, both palaeontologically and molecularly [18] identified. SH-Hsnn inhabited northern Spain, while $H s n n^{*}$ reigned both in Europe and Asia, as established by extensive Eurasian fossil finds.

The arrowheads that lead from $H s s$ to $H s n n^{*}$ in Fig. 1 mark the phylogeny resulting from the mtDNA introgression that took place from $H s s$ into $H s n n^{*} \approx 500,000$ YBP [1 As shown in Fig. 2 the introgression joins $H s s$ and $H_{s n n^{*}}$ on a common mtDNA branch, therewith restricting the initial mtDNA branch of Hsn to SH-Hsnn and Hsnd. With the Neanderthals strictly limited to Eurasia it becomes apparent that the mtDNA introgression from $H s s$ to $H s n n^{*}$ could only take place in conjunction with the contemporary coexistence of both Hss and Hsnn in Eurasia $[1,6]$, a circumstance that invalidates OOAH since that hypothesis does not allow the existence of $H s s$ in Eurasia at the time in question.

An extensive genomic study of extant humans that was presented a few years ago [20] identified a basal Hss divergence between a Eurasian (French) genome and the genome of the African Mbuti, but the significance of the finding for addressing Hss origin and evolution was not discussed. However, as maintained subsequently on phylogenetic grounds [6], this basal Hss divergence compromised OOAH since that hypothesis rested instead upon an $H s s$ exodus out of Africa into Eurasia by a late arising African population and not on a population that constituted the earliest divergence among recent humans.

In the recent study by Árnason and Hallström [1] the direction of evolution in the Hss tree was established by applying a new approach, Progressive Phylogenetic Analysis, PPA, which demonstrated that the African populations constituted a paraphyletic grouping. The African paraphyly invalidated the postulate of an $H s s$ origin in Africa and a late exodus out of that continent into Eurasia since the PPA identified instead a minimum of three separate waves of $H s s$ migration from Eurasia into Africa the earliest being that of the ancestors of Mbuti/San. Thus, in 
contradiction to OOAH the PPA approach yielded results that were consistent with a continuous unidirectional Eurasian evolution on the $H s$ branch from the divergence between $H$. antecessor and $H$. sapiens to the apical tips of extant $H s s$ as demonstrated in Figs. 1 and 2.

\section{The profiles of the Y-DNA and mtDNA phylogenies of Hss}

Figure $3 \mathrm{a}-\mathrm{c}$ shows the phylogenetic relationships of recent $H s s$ as resolved in analysis of two separate sets of data, the paternally transmitted Y-DNA and the maternally transmitted mtDNA. The Y-DNA tree of OOEH $[3,4]$ is shown to the left in the figure, the mtDNA tree of OOEH in the middle [1] and the commonly acknowledged Y-DNA tree of OOAH (e.g. [2]) to the right. Although the three trees are superficially similar they are fundamentally different in that trees $3 \mathrm{a}$ and $3 \mathrm{~b}$ are consistent with the out of Eurasia hypothesis whereas tree 3c is that of OOAH.

The extensive Y-DNA study [4] behind Fig. 3a showed a minimum of five waves of Hss migration from Eurasia into Africa while the more limited mtDNA sampling behind $3 \mathrm{~b}[1]$ identified a minimum of three migrations with each of these coinciding with the Y-DNA results, consistent with the presence of both males (Y-DNA) and females (mtDNA) in each population migrating into Africa. As apparent a divergence between Mbuti and San prior to their migration into Africa [5] would raise the number of waves into Africa by one, as would also any migration connected to the $\mathrm{B}+\mathrm{C}$ branch [4] that has been tentatively indicated on the mtDNA tree.

With respect to the phylogeny in Fig. 3c it should be noted that the OOAH tree and the out of Africa hypothesis, in addition to their earlier rebuttal [3, 4], became rejected by the recent PPA findings [1] which demonstrated the paraphyly of the African populations. In contradiction to OOAH these molecular results were all in accordance with the out of Eurasia hypothesis and the Eurasian palaeontology of both Hss and $H s n(H s n n+H s n d)$, a topic that has been largely ignored by the adherents of OOAH.

The Y-DNA tree in Fig. 3a and the mtDNA tree in 3b are both consistent with a unidirectional Hss evolution from the Eurasian root of the $H s$ tree to the tip of each individual $H s s$ branch. The Y-DNA tree is based upon the largest sample that has been used to delineate the Y-DNA relationships of extant humans. The analysis [4] identified a Eurasian Y-DNA coalescence, A00, that was followed by three separate $H s s$ exoduses, A0, A and $\alpha$, from Eurasia into Africa with A0 signifying the earliest and $\alpha$ the most recent of these early exoduses. The Eurasian Y-DNA phylogeny shows a continuous Eurasian span from position $\alpha$ to the extensive Eurasian diversification beginning at position $\beta$ in the Y-DNA tree.

The mtDNA tree in Fig. 3b mirrors the Y-DNA phylogeny in accordance with a shared identity, male and female, within each pair of the Hss exoduses from Eurasia into Africa. Similarly the barren Eurasian branch between

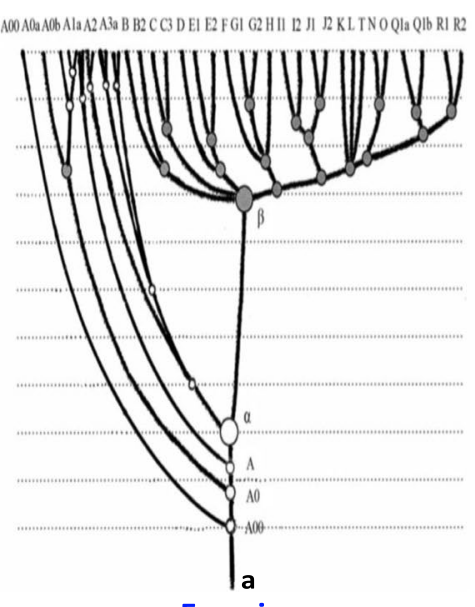

Eurasia

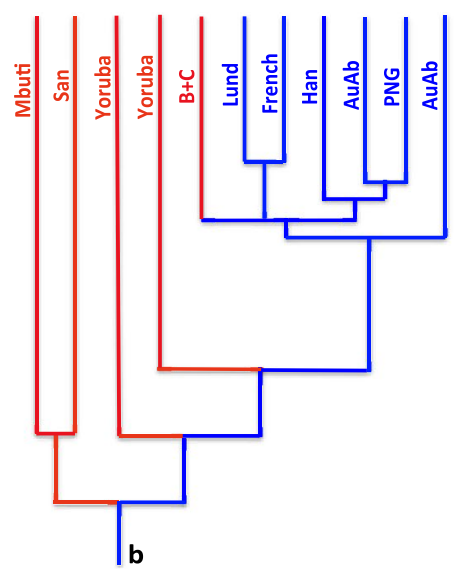

Eurasia

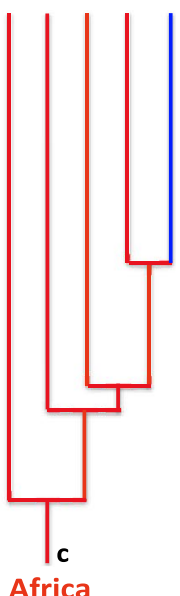

Africa

Fig. 3 The Y-DNA and mtDNA phylogenies of Hss with the Y-DNA tree (a) [4] and the mtDNA tree (b) [1] representing the OOEH phylogeny, and tree (c) standing for the OOAH phylogeny of both mtDNA and Y-DNA. Trees (a) and (b) are consistent with a residing Eurasian Hss populations and a series of Hss exoduses from Eurasia into Africa. Position A00 in the Y-DNA tree and the corresponding position in the mtDNA tree mark the position at which the basal African and non-African lineages of extant Hss populations coalesce. Position $\beta$ in tree (a) signifies the beginning of the Eurasian diversification of Hss dated to $\approx 125,000$ to 120,000 YBP in the mtDNA tree [1]. The blue branch in (c) signifies a late Hss exodus out of Africa as assumed by the out of Africa hypothesis. As underlined in Figs. 1 and 2, the position of the root of the phylogeny in tree (c) is without connection to the Eurasian evolution of Hss and Hsn(Hsnn +Hsnd) 
the last exodus into Africa, and position $\beta$, the initiation of the Eurasian diversification of $H s s$ is common to both phylogenies although the span is longer in the Y-DNA phylogeny than in the mtDNA tree, a distinction that might be related to different modes of calculation.

The phylogenetic position at position $\beta$ in the Y-DNA tree was discussed in the recent mtDNA study [1] in the context of climatic cycles, the most severe of these ending $\approx 125,000$ YBP. This climatic circumstance coincides with the restricted molecular variation at the corresponding position in the Eurasian phylogeny prior to the striking population expansion occurring later in the two separate sets of molecular data behind the Y-DNA and mtDNA phylogenies.

\section{Conclusions}

The Y-DNA and mtDNA phylogenies discussed here are consistent with a unidirectional $H s$ evolution from the Eurasian root of the $H s$ tree to each individual branch connected to Hss evolution, including the populations that migrated from Eurasia into Africa. The findings compromise the large number of results that have been interpreted in accordance with the Out of Africa hypothesis and the a priori assumption that the African Hss populations constituted a monophyletic assembly from which Eurasia became colonized. A crucial phylogenetic circumstance related to the rebuttal of OOAH has been the identification of the separate $H s s$ exoduses into Africa with each of these recorded by both the female inherited mtDNA and the male inherited Y-DNA.

\section{Acknowledgements \\ The author thanks Prof. Eric H. Harley, University of Cape Town, RSA, for valu- able comments and suggestions and Prof. Anatole A. Klyosov, Harvard Medical School, MA, USA, for Fig. 3a. The author also thanks The Erik Philip-Sörensens Foundation and The Royal Physiographic Society, Lund, for support.}

\section{Author's contributions}

The author read and approved the final manuscript.

\section{Declarations}

\section{Competing interests}

The author declares that he/she has no competing interests.

\section{Author details}

'Department of Clinical Sciences Lund, Lund University, Lund, Sweden. ${ }^{2}$ Department of Neurosurgery, Skane University Hospital in Lund, Lund, Sweden

Received: 10 June 2021 Accepted: 10 August 2021

Published online: 14 September 2021
References

1. Árnason Ú, Hallström B. The reversal of human phylogeny: Homo left Africa as erectus, came back as sapiens sapiens. Hereditas. 2020;157:51. https://doi.org/10.1186/s41065-020-00163-9.

2. Scozzari R, et al. Molecular dissection of the basal clades in the human Y chromosome phylogenetic tree. PLoS One. 2012;7(11):1-5.e49170 https://doi.org/10.1371/journal.pone.0049170.

3. Klyosov AA, Rozhanskii IL. Re-examining the "Out of Africa" theory and the origin of Europeoids (Caucasoids) in light of DNA genealogy. Adv Anthropol. 2012;2:80-6. https://doi.org/10.4236/aa.2012.22009.

4. Klyosov AA. Reconsideration of the "Out of Africa" concept as not having enough proof. Adv Anthropol. 2014;4:18-37. https://doi.org/10.4236/aa. 2014.41004.

5. Árnason Ú. The out of Africa hypothesis and the ancestry of recent humans: cherchez la femme (et l'homme). Gene. 2016;585:9-12.

6. Árnason Ú. A phylogenetic view of the out of Asia/Eurasia and out of Africa hypotheses in the light of recent molecular and palaeontological finds. Gene. 2017:627:473-6.

7. Cann RL, Stoneking M, Wilson AC. Mitochondrial DNA and human evolution. Nature. 1987:325:31-6.

8. Vigilant L, Stoneking M, Harpending H, Hawkes K, Wilson AC. African populations and the evolution of human mitochondrial DNA. Science. 1991;253:1503-7.

9. Maddison DR. African origin of human mitochondrial DNA reexamined. Syst Zool. 1991;40:355-63.

10. Templeton AR. Human origins and analysis of mitochondrial DNA sequences. Science. 1992;255:737.

11. Hedges BS, Kumar S, Tamura K, Stoneking M. Human origins and the analysis of mitochondrial DNA sequences. Science. 1992;255:737-9.

12. Petr M, Hajdinjak M, Fu Q, Essel E, Rougier H, Crevecoeur I, et al. The evolutionary history of Neanderthal and Denisovan Y chromosomes. Science. 2020;369:1653-6.

13. Posth C, Wißing C, Kitagawa K, Pagani L, van Holstein L, Racimo F, et al. Deeply divergent archaic mitochondrial genome provides lower time boundary for African gene flow into Neanderthals. Nat Commun. 2017. https://doi.org/10.1038/ncomms16046.

14. Meyer M, Arsuaga J-L, de Filippo C, Nagel S, Aximu-Petri A, Nickel B, et al. Nuclear DNA sequences from the Middle Pleistocene Sima de los Huesos hominins. Nature. 2016;531:504-7.

15. Zhu Z, Dennell R, Huang W, Wu Y, Qiu S, Yang S, et al. Hominin occupation of the Chinese loess plateau since about 2.1 million years ago. Nature. 2018;559:608-12.

16. de Castro JMB, Arsuaga JL, Carbonell E, Rosas A, Martínez I, Mosquera M. A Hominid from the Lower Pleistocene of Atapuerca, Spain: possible ancestor of Neandertals and modern humans. Science. 1997;276:1392-5.

17. Welker F, Ramos-Madrigal J, Gutenbrunner P, Mackie M, Tiwary S, JersieChristensen RR, et al. The dental proteome of Homo antecessor. Nature. 2020;580:235-8.

18. Arnason U, Xu X, Gullberg A. Comparison between the complete mitochondrial DNA sequence of Homo and the common chimpanzee based on nonchimeric sequences. J Mol Evol. 1996:42:145-52.

19. Meyer M, Fu Q, Aximu-Petri A, Glocke I, Nickel B, Arsuaga J-L, et al. A mitochondrial genome sequence of a hominin from Sima de los Huesos. Nature. 2014;505:403-5.

20. Mallick S, Li H, Lipson M, Mathieson I, Gymrek M, Racimo F, et al. The Simons genome diversity project: 300 genomes from 142 diverse populations. Nature. 2016;538:201-6.

\section{Publisher's Note}

Springer Nature remains neutral with regard to jurisdictional claims in published maps and institutional affiliations. 